Bull. Mater. Sci., Vol, 17, No. 6, November 1994, pp. 717-732. (O Printed in India.

\title{
A unified approach to complex crystalline and so-called quasicrystalline phases in aluminium alloys*
}

\author{
T R ANANTHARAMAN \\ Metals and Alloys Group, National Physical Laboratory, Dr K S Krishnan Road, New \\ Delhi 110 012, India
}

\begin{abstract}
Recent developments in the complex and controversial, but fertile and fascinating field of aluminium-transition metal alloys are reviewed with particular reference to the so-called quasicrystalline phases and their rational approximants. Pauling's several approaches to icosahedral phases on the basis of twinning in cubic crystals with large unit cells are described and examined along with the difficulties in checking the structures proposed by him. A new unified crystallographic approach is presented by the author, starting with icosahedral atomic clusters in concerned alloy melts, and it is shown that this integral approach leads to a reasonably good understanding of the interrelationship between the numerous known solid phases in aluminium alloys, including icosahedral phases, decagonal phases and their rational approximants.
\end{abstract}

Keywords. Complex crystalline phases; quasicrystalline phases; aluminium alloys.

\section{Introduction}

Aluminium ( $\mathrm{Al}$ ) is today commercially the second most important metal in the world, even though a rather distant second to iron ( $\mathrm{Fe}$ ) which accounts for over $90 \%$ of the global annual output of metals and alloys. The tremendous technological importance of aluminium stems from its lightness, high electrical and thermal conductivity, excellent corrosion resistance, easy workability and attractive mechanical propert, , s on alloying. No wonder, therefore, that aluminium alloys have been under study by che mists, crystallographers and metallurgists for many decades now. It became clear even by the fifties of this century (Bland 1958) that the crystal structures of intermetallic phases based on aluminium tend to be complex with much larger unit cells than associated with metals and alloys in general. The Frank-Kasper phases, e.g. $(\mathrm{AlZn})_{49} \mathrm{Mg}_{32}$ and $\mathrm{Al}_{6} \mathrm{Mg}_{4} \mathrm{Cu}$, with 162 atoms in the unit cell, and Samson's $\mathrm{Al}_{3} \mathrm{Mg}_{2}$ phase with 1168 atoms in the unit cell have attracted special attention in this regard (see Shoemaker and Shoemaker 1988). Apart from these thermodynamically stable, equilibrium phases, a number of metastable, non-equilibrium phases including metallic glasses (see Anantharaman 1984) could be produced by rapid solidification (see Anantharaman and Suryanarayana 1987) in binary and ternary aluminium alloys during the last three decades. All these phases were amorphous or crystalline and the atomic arrangements in them could be explained and understood in terms of traditional ideas and concepts developed during the first half of this century on the structure of solids.

However, the scientific world was in for a great surprise almost exactly a decade ago when a team of Israeli, French and American investigators (Schechtman et al

*DEDICATED TO THE MEMORY OF PROFESSOR LINUS PAULING (1901-1994). 
1984) reported on the occurrence of crystallographically forbidden five-fold diffraction symmetry in rapidly solidified Al-Mn alloys. These researchers asserted in their report that the $\mathrm{X}$-ray diffraction pattern from this icosahedral phase, made up of a few strong and medium and very many weak and very weak reflections, 'could not be indexed to any Bravais lattice' and further, multiple twinning capable of explaining the observed icosahedral symmetry was ruled out on the evidence of 'dark-field images from any reflection' illuminating 'in all cases the whole grain' in electron micrographs. These bold assertions were received enthusiastically and accepted readily by most physicists and chemists interested in structural problems without any critical examination or comprehensive debate of the complex metallurgical issues involved. Thus began what looked like a new and exciting era in solid-state sciences based on the fascinating and novel paradigm of 'quasicrystallinity', postulated a little earlier for a possible new species of solids (Levine and Steinhardt 1984) through three-dimensional analogs of Penrose tiling (Mackay 1982). Concept after concept, model after model, review after review, conference after conference, book after book-all followed in quick succession, accounting for over 3000 papers on the quasicrystal phenomenon from all parts of the globe in less than a decade! All the same, despite the intense research efforts, often multi-institutional and multi-national, on the part of numerous physicists, mathematicians and materials scientists, all directed towards a few dozen icosahedral and decagonal phases (see table 1), most of them metastable and produced by rapid solidification of aluminium-transition metal (Al-TM) binary and ternary alloys, the exact atomic arrangement has not been established to this day for any one of these exotic phases on the basis of quasicrystallography.

A new dimension was added to the quasicrystal saga in 1988 with the production of thermodynamically stable quasicrystalline phases by normal cooling from the melt, like the Al-Cu-Fe, Al-Cu-Ru and Al-Pd-Mn icosahedral phases, as also the

Table 1. Approximate compositions of typical icosahedral and decagonal phases based on aluminium.

\begin{tabular}{ll}
\hline Icosahedral phases & Decagonal phases \\
\hline $\mathrm{Al}_{82} \mathrm{Mn}_{18}$ & $\mathrm{Al}_{80} \mathrm{Mn}_{20}$ \\
$\mathrm{Al}_{85} \mathrm{Cr}_{15}$ & $\mathrm{Al}_{78} \mathrm{Co}_{22}$ \\
$\mathrm{Al}_{86} \mathrm{Fe}_{14}$ & $\mathrm{Al}_{80} \mathrm{Ni}_{20}$ \\
$\mathrm{Al}_{80} \mathrm{Ru}_{20}$ & $\mathrm{Al}_{80} \mathrm{P}_{20}$ \\
$\mathrm{Al}_{80} \mathrm{~V}_{20}$ & $\mathrm{Al}_{80} \mathrm{Os}_{20}$ \\
$\mathrm{Al}_{78} \mathrm{Re}_{22}$ & $\mathrm{Al}_{80} \mathrm{Fe}_{20}$ \\
$\mathrm{Al}_{60} \mathrm{Li}_{30} \mathrm{Cu}_{20}$ & \\
$\mathrm{Al}_{62} \mathrm{Cr}_{19} \mathrm{Si}_{19}$ & \\
$\mathrm{Al}_{73} \mathrm{Mn}_{21} \mathrm{Si}_{6}$ & $\mathrm{Al}_{79} \mathrm{Mn}_{19} \mathrm{Fe}_{2}$ \\
$\mathrm{Al}_{60} \mathrm{Ge}_{20} \mathrm{Mn}_{20}$ & $\mathrm{Al}_{65} \mathrm{Cu}_{20} \mathrm{Mn}_{15}$ \\
$\mathrm{Al}_{70} \mathrm{Fe}_{20} \mathrm{Ta}_{10}$ & $\mathrm{Al}_{65} \mathrm{Cu}_{20} \mathrm{Co}_{15}$ \\
$\mathrm{Al}_{65} \mathrm{Cu}_{20} \mathrm{Fe}_{15}$ & $\mathrm{Al}_{75} \mathrm{Cu}_{10} \mathrm{Ni}_{15}$ \\
$\mathrm{Al}_{65} \mathrm{Cu}_{20} \mathrm{Ru}_{15}$ & $\mathrm{Al}_{60} \mathrm{Cu}_{25} \mathrm{Co}_{15}$ \\
$\mathrm{Al}_{65} \mathrm{Cu}_{20} \mathrm{Os}_{15}$ & $\mathrm{Al}_{63} \mathrm{Cu}_{17.5} \mathrm{Co}_{17.5} \mathrm{Si}_{2}$ \\
\hline
\end{tabular}


$\mathrm{Al}-\mathrm{Cu}-\mathrm{Co}$ and $\mathrm{Al}-\mathrm{Cu}-\mathrm{Co}-\mathrm{Si}$ decagonal phases, all of them displaying extremely sharp X-ray and neutron reflections, as would be expected of normal annealed crystalline metals (see Steurer 1990). The availability of single crystals of these phases has facilitated high-resolution studies using powerful synchrotron radiation during the last few years (Motsch et al 1992; Fettweis et al 1993). However, these studies have only thrown up further questions concerning the very validity of the quasicrystalline concept. As conceded recently by an acknowledged leader of the quasicrystallography school (Janot 1993) 'quasicrystallography has not yet reached the level of structure refinement that is currently reported in regular crystallography. Also the description of the structure though very easy in its high-dimensional periodic image, is not that straightforward when one deals with the three-dimensional physical atomic arrangements'. Further, as highlighted by two other strong advocates of quasicrystallography (Goldman and Kelton 1993), a second fundamental and ticklish issue has arisen, relating to 'the limits of our ability to distinguish through experiment between quasicrystals and multiply-twinned crystals with large unit cells'. Yet another significant development has been the discovery, one after the other, of many so-called rational crystalline approximants of quasicrystalline. phases, which show pseudo-five-fold symmetry and are very difficult to distinguish and differentiate from the corresponding icosahedral or decagonal phases (Liu 1993; Anantharaman 1994).

\section{Crystallinity and icosahedral motifs}

Shortly after publication of the above-referred first report on the Al-Mn icosahedral phase alternative crystallographic approaches to these phases were advocated independently by Field and Fraser (1985), Carr (1985) and Pauling (1985). Multiple twinning or icosatwinning was implicit in all these approaches and not surprisingly, while striking a sympathetic chord among the crystallographic community (Shoemaker and Shoemaker 1988), they drew emotionally surcharged and aggressively adverse comments-dead cat and thriving pigeons!-from the pioneers of quasicrystallography (Bancel et al 1986; Cahn et al 1986; Mackay 1986). By the late eighties, despite reservations from chemists and metallurgists, the condensed-matter physicists and mathematicians seemed to have made their choice overwhelmingly in favour of the new quasicrystal paradigm (Shoemaker and Shoemaker 1988) revolving round jargons like six-dimensional space, strip projection, a-periodicity, Fibonacci sequence, phason strain etc as also models like three-dimensional Penrose tilings, random packing of icosahedral clusters, icosahedral glasses, entropic quasicrystals etc. All the same, the traditional crystallographic framework was invoked repeatedly and developed further by Pauling (1987a,b,c, 1988a-d, 1989a,b, 1990, 1991) and the present author (Anantharaman 1987, 1988a,b, 1989, 1990, 1993, 1994), chemist and metallurgist respectively, despite a uniform and persistent tendency on the part of most quasicrystallographers to ignore any suggestion of a possible crystallographic solution to this problem.

It is necessary to record in this context that chemists and metallurgists have known for some decades now that local icosahedral arrangements of atoms are possible and, in fact, do occur in a large number of inter-metallic phases, particularly in Al-TM alloys. Such icosahedral groupings have local and approximate icosahedral 
symmetry. It should be clear to any one familiar with the whole field of complex crystalline phases based on aluminium, particularly those containing transition metals with smaller atoms than those of the base metal, that icosahedral groups and linkages thereof are bound to constitute important features of the new quasicrystalline phases as well. Support to this view is lent by two facts viz.'some icosahedral phase compositions are the same (e.g. Frank-Kasper phases) or approximately the same as the compositions of known crystalline alloys and there are generally striking correspondences between the two in observed diffraction intensities (Shoemaker and Shoemaker 1988). In fact, even quasicrystallographers must surely have caught glimpses of what strikes any impartial observer as some sort of basic unity that underlies and connects up different phases displaying ideal or distorted icosahedral motifs.

As early as in 1987 the present author made the following concluding observations in a keynote address at the Beijing International Conference on Quasicrystals: 'An important consideration here is the close inter-relationship between the liquid, glassy quasicrystalline and in some cases even crystalline (e.g. Frank-Kasper) phases in some alloy systems. Obviously a unified approach is called for that can explain this closeness in atomic arrangements between three or four different phases with the same alloy composition. If icosahedral clusters or chains do exist in metallic liquids-the evidence so far seems to be in favour-they will have to be the starting point in model making' (Anantharaman 1987). Starting from 1988, Pauling has been referring in his papers to a 'unified structure theory' for crystalline and quasicrystalline phases based on icosahedral atomic clusters that 'can be expected to be present in molten alloys' made up of two or more metallic elements with different atomic radii (Pauling 1988a-d, 1989a,b).

An attempt is made in this paper to look for and identify the unifying link, the golden thread, as it were, that connects different Al-TM phases, with the author's model for quasicrystalline phases and their rational approximants (Anantharaman 1993, 1994) serving as a possible starting point for the quest.

\section{Quasicrystals or complex crystals?}

An objective assessment of the facts and findings enumerated above is bound to lead to the conclusion that the time has definitely come for the scientific community to compare comprehensively and evaluate critically the quasicrystalline and crystalline approaches to icosahedral and decagonal phases in aluminium alloys. As recorded above, the extreme complexity and unusual features of many equilibrium phases in Al-TM systems have been known and recognized as such by crystallographers for nearly half a century. These phases generally feature prominent layers or configurations of transition metal atoms, the latter generally avoiding each other in ternary and quaternary phases (Bland 1958). They also display a zone of strong $X$-ray reflections corresponding to interplanar spacings of about $0.2 \mathrm{~nm}$, around where the 111- and 200-reflections of aluminium occur. As for non-equilibrium phases of these alloy systems, produced generally by rapid quenching of metallic melts, they are characterized by complexity of structure, twinning, stacking faults, small domain sizes etc (Anantharaman et al 1977). Unfortunately, most quasicrystallographers have not paid sufficient attention to the abundant 
crystallographic structural data that have thus been amassed over decades for aluminium alloys of compositions similar or related to those of the new quasicrystalline phases.

Over the years Pauling and the present author have drawn the attention of the scientific community to several experimental facts which are not explained by or go contrary to the quasicrystallographic approach to icosahedral phases:

(i) Much has been made from the beginning of dark-field images for different reflections illuminating the entire grain in electron micrographs. The fact has been conveniently overlooked that the entire grain is never uniformly and identically illuminated from each imaging-in fact, there is always a sub-structure strongly suggesting the presence of nanometer-level micro-crystals with different orientations (Anantharaman 1988a).

(ii) Despite the early assertions that the $\mathrm{X}$-ray diffraction patterns of icosahedral phases cannot be indexed to any Bravais lattice, cubic and orthorhombic unit cells have been proposed (Pauling 1987a,b,c; Anantharaman 1989) and satisfactory agreement between observed and calculated interplanar spacings also demonstrated for many icosahedral phases.

(iii) Many deviations from ideal quasicrystallography have been noted in electron diffraction patterns. The ten spots in any circle are generally identical neither in shape nor in intensity (Anantharaman 1988a) and many spots seem to be due to overlap of two or more reflections (Vecchio and Williams 1988). Measurement of angles between rows of reflections shows deviations from the expected $36^{\circ}$, while positions of diffraction maxima in any row of reflections show deviations from the expected Fibonacci sequence (Pauling 1989a,b).

(iv) The positions and shapes of some electron diffraction spots strongly suggest that they are formed by double diffraction (Pauling 1989a,b). In fact, a detailed comparison of Debye-Scherrer and electron-diffraction patterns of the equilibrium Al-Cu-Fe icosahedral phase has recently confirmed (Anantharaman 1994a) the role of double diffraction in the latter. This study has also established that the observed five-fold diffraction symmetry in such phases is due to the special geometry of their unit cells and the co-existence of five sets of micro-crystals with a special $72^{\circ}$ orientation relationship between them in every grain or single crystal of these phases.

(v) More than one orientation has actually been observed in high-magnification electron micrographs of icosahedral phases. Ball and Lloyd (1985) reported on the appearance of all five orientations in the $\mathrm{Al}_{6} \mathrm{Li}_{3} \mathrm{Cu}$ phase, while Rao and Anantharaman (1988) could detect three or four orientations $72^{\circ}$ to each other in the $\mathrm{Al}-\mathrm{Cu}-\mathrm{Mg}$ phase through lattice resolution or Moiré fringes.

(vi) The non-icosahedral, micro-crystalline nature of the $\mathrm{Al}-\mathrm{Li}-\mathrm{Cu}$ quasicrystalline phase was also brought out effectively by Vecchio and Williams (1988) through convergent-beam electron diffraction (CBED) studies. Asymmetries observed in CBED patterns showed that the crystal symmetry is not truly five-fold. By careful thinning of the specimen to a wedge-like shape, the twinned micro-crystalline nature of the phase was brought out by these investigators in a striking fashion.

The most clinching evidence in favour of traditional crystallography and against quasicrystallography has just been put on record by a French team (Fettweis et al 1994) which has been quite enthusiastic about the quasicrystal concept until two 
years ago. Analysing systematically their single crystal $\mathrm{X}$-ray diffraction data from decagonal $\mathrm{Al}_{63} \mathrm{Cu}_{17.5} \mathrm{Co}_{17.5} \mathrm{Si}_{2}$, these researchers come to the following conclusion: 'High resolution experiments using synchrotron radiation are well interpreted within the scope of a micro-crystalline model. The decagonal needles are in a micro-crystalline state formed of crystalline domains having $72^{\circ}$ orientation relations'.

In the light of the above findings, it is appropriate to examine in some detail the available two crystallographic models proposed and developed over the years by Pauling and the present author respectively for quasicrystals in general and icosahedral phases in particular.

\section{The Pauling hypothesis}

As is well recognized, Pauling and his collaborators used the stochastic method of trial-and-error with educated guesses (Pauling 1955) very frequently and highly successfully since the fifties of this century in the formulation of structure models for complex inter-metallic phases, particularly in aluminium alloys. Using the same method, Pauling studied X-ray and electron diffraction patterns of icosahedral phases obtained from other investigators and came out with many proposals of cubic unit cells that can lead to five-fold diffraction symmetry through icosa-twinning. He proposed first a 1168-atom cubic unit cell with $a=26.73 \AA$ on the pattern of $\mathrm{NaCd}_{2}$ (Pauling 1985) for Al-Mn icosahedral phase (i-Al-Mn). Realizing that the sequence of electron diffraction maxima in the five-fold patterns could not be satisfactorily explained with such a cubic cell on the basis of twinning, he modified his unit cell to one with 820 atoms and $a=23.36 \AA$ (Pauling 1987a,b,c), made up of eight 104-atom clusters, each being a complex of twenty Friauf polyhedra with icosahedral symmetry. According to this model, the eight 104-atom complexes adopt the $\beta-W$ structure i.e. two complexes at 000 or $1 / 21 / 21 / 2$ positions and six complexes at $1 / 2 \pm 1 / 40, \pm 1 / 41 / 20$ and $01 / 2 \pm 1 / 4$ positions in the large unit cell, with twelve atoms lost in the process of sharing of pairs $(8 \times 104-12)$. Incidentally, such clusters of 104 atoms are known to occur in $(\mathrm{AlZn})_{49} \mathrm{Mg}_{32}$ and other Frank-Kasper phases (Bergman et al 1957).

Following availability of reliable X-ray powder and electron diffraction patterns from equilibrium icosahedral phases like $i-\mathrm{Al}-\mathrm{Cu}-\mathrm{Fe}$, Pauling examined their very many weak and very weak $\mathrm{X}$-ray reflections, particularly at very low Bragg angles, 'which are ten times as numerous as the stronger peaks' (Pauling 1988a-d) as also the distribution of spots in two-fold axis electron diffraction patterns (Pauling $1989 \mathrm{a}, \mathrm{b})$ and came to the conclusion that much larger cubic unit cells were necessary to explain the available X-ray and the electron data. Thus he proposed a body-centred cubic cell with the cube edge value around $52 \AA$ and containing over 9,900 atoms for $i-\mathrm{Al}-\mathrm{Cu}-\mathrm{Fe}$ and an even bigger face-centred cubic unit cell ( $a=66 \AA$ with over 19,000 atoms!) for $i$-Al-Mn (Pauling 1989a,b). He invoked the $\beta$-W arrangement again for $i$-Al-Cu-Fe, but with very large complexes, each an icosahedral packing $\begin{array}{lllll}\text { made up of no less than forty-five 104-atom clusters at } 000 \text { and } 1 / 2 \quad 1 / 2 & 1 / 2\end{array}$ positions, and individual 104-atom clusters in the other six positions.

Pauling based his work on experimental evidence from others and often could not (or did not) index all $X$-ray reflections on the basis of his unit cells. Nor did he explain why so many expected reflections were not observed. The agreement 
between observation and calculation was not always satisfactory and the indices $(h k l)$ of strong or medium reflections were sometimes far from convincing e.g. 13.85, $21 \cdot 13.8,34.21 \cdot 13$ in case of $i$-Al-Cu-Fe. Although a space group viz. No. 233, was assigned to this cubic unit cell, no effort was made to show matching of observed X-ray intensities with calculated values. In his own words (Pauling 1991) such structures are, 'of course, hypothetical at the present time' ... 'It is not possible at the present time to check this proposed structure and locate the atoms'.

However, Pauling was all along convinced that his proposals were heading to a 'unified structure theory' for thermodynamically stable as well as non-equilibrium metastable phases in aluminium alloys. By invoking cubic crystallites with structures closely similar to those of known intermetallic compounds for the new icosahedral phases, he strongly felt that his unified theory 'has the desirable feature of conforming to the known principles of structural chemistry'. One cannot do better than quote Pauling himself on his vision of a unified structure theory (Pauling 1988a-d):

'The formation of large icosahedral clusters is expected for an alloy of two or more metallic elements with different atomic radii, and these clusters, probably with a range of values of the number of atoms in the outer shell, can be expected to be present in the molten alloy. If the molten alloys were to be quenched extremely rapidly it would form a metallic glass, with the clusters having the same random orientation as in the melt and being piled together as in the melt. With somewhat less rapid quenching the clusters would begin to form crystallites. These crystallites might in some cases, as for $\mathrm{Al}_{6} \mathrm{CuLi}_{3}$, have the thermodynamically stable structure. Usually, however, as for $(\mathrm{AlZn})_{49} \mathrm{Mg}_{32}$, the thermodynamically stable structure involves sharing the atoms in the outer shell between clusters, which requires time, so that instead the alloy crystallizes with a metastable structure that involves an easily assumed arrangement in which the clusters that were present in the liquid pile together in a crystallographically simple way, such as cubic close packing of the clusters, 4 clusters in a face-centred cube.

In fact, no such 4-cluster quasicrystals have been found; instead, the structures are based on the $\beta-W$ arrangement, with the unit cube containing 8 clusters. The reason for the selection of $\beta-W$ arrangement is obvious. It is that the Bergman-Waugh-Pauling triple-shell cluster of 104 to 136 atoms has icosahedral point group symmetry and accordingly strives to coordinate 12 other clusters about itself at the corners of the icosahedron .... The diffraction maxima in these grains have intensities and positions determined by three structure factors: the structure factor of the cluster, the structure factor of the cubic crystallite and the structure factor representing the interaction of one crystallite with the other'.

\section{The author's model}

The present author has been studying carefully and systematically since $1987 \mathrm{X}$-ray powder and electron diffraction patterns from icosahedral and decagonal phases, some of them obtained from other investigators and some generated by him in 
Indian laboratories (at Varanasi, Patiala and Delhi) or elsewhere during short visits (Tokyo in 1987, Oxford in 1989, Grenoble in 1990, Dortmund in 1993 and Dresden in 1994). He has thereby been trying to understand the atomic arrangements in these new and exotic phases in his capacity as a structural metallurgist operating within the framework of traditional crystallography. His objective has been not only to understand and explain the observed diffraction effects satisfactorily, but also to propose and develop a model that can account for all experimental observations related to the formation and transformation as also physical, chemical and mechanical properties, of these phases and relate them to other equilibrium as well as non-equilibrium phases in aluminium alloys. In view of the universal observation of icosahedra, icosahedral order or icosahedral motifs in practically all complex Al-TM alloy phases, the starting point for the author's model has been the icosahedral atomic cluster suggested by Frank (1952) and later expanded to include pentagonal atomic chains through vertex connection of icosahedra by Hume-Rothery and Anderson (1960). It is of some interest to record here that two of the earlier models put forward by quasicrystallographers themselves were based on icosahedra either joined randomly by their edges (Schechtman and Blech 1985) or connected through their vertices with bond-orientational order (Stephens and Goldman 1986).

As has been lucidly brought out by Shoemaker and Shoemaker (1988), chemical crystallographers have long been familiar with the variety of ways in which icosahedra can link together to propagate the icosahedral orientation through space. As shown in figure 1 two icosahedra can join or get welded through sharing a vertex (figure 1a), or an edge (figure 1b) or a face (figure 1c), with two types of orientations ( $A$ and $B$ ) emerging, the orientation $B$ derived from orientation $A$ by the operation of a mirror plane. The three modes of linking separated icosahedra are brought out in figure 2 , through intervening tetrahedra (figure 2a), or octahedra (figure 2b) or squares made up of opposite edges (figure $2 \mathrm{c}$ ). Needless to say, despite minor distortions in the joining process, the shapes and sizes of unit cells of the resulting crystal structures get determined and hence may be computed on the basis of the icosahedron's geometry, as modified by requirements of the concerned linkages.

The primary building block for icosahedral and decagonal phases, as also their many rational approximants, is identified in the author's model (Anantharaman

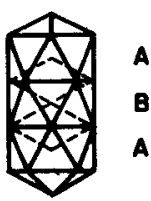

(a)

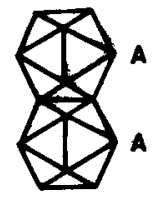

(b)

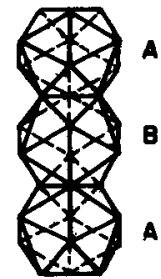

(c)

Figure 1. Mlocks of jouning icosahedra: (a) two icosahedra (both having orientation A) sharing vertex to generate a third icosebedron in between with orientation $B$, (b) two icosahedra sharing an edge and (c) icosahedra sharing a face with orientations alternating. 
1993, 1994) as the orthorhombic cell (figure 3) that can be visualized as forming through linkage in three perpendicular directions, with small expansion, contraction and/or distortion, as may be required, of icosahedral clusters containing more than one species of metallic atoms. Following atom-sharing in this joining process involving all three connections described above (figures $3 a, b$ and $c$ ), this basic orthorhombic cell (actually a distorted icosahedron) ends up with 6 or 7 effective atoms in it, the latter number in case of filled icosahedra with 13 atoms, one of them in the body centre. This basic cell can be assigned the space group $P_{\mathrm{mmm}}$ (No. 47) and provided 16 possible atomic positions for the 6 or 7 effective atoms in it, with an unusual face-centred-cum-body-centred-cum-edge-centred symmetry that facilitates ordering of different atoms and vacancies in diverse ways in superstructures. In fact, this unit cell can be halved as shown in figure 4a, with 8 atomic positions for 3 to 3.5 effective atoms, the former characterized by two variants of face-centred cubic orthorhombic positions that enable maintenance of appropriate inter-atomic distances, partial occupancy of atomic sites and vacancies at required levels in superstructures.

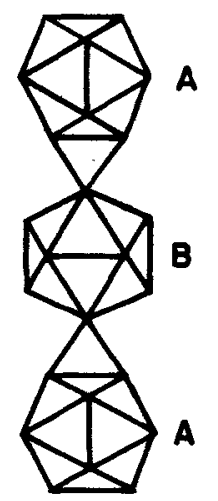

(a)

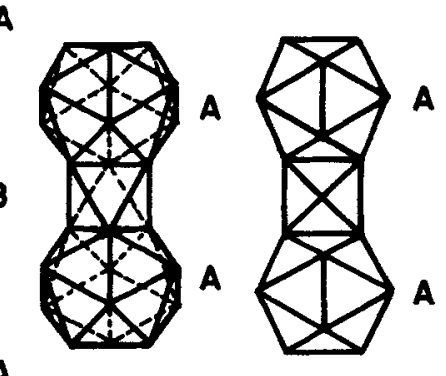

(b)

(c)

Figure 2. Modes of linking separate icosahedra: (a) icosahedra join, sharing opposite edges with tetrahedra that separate them, (b) icosahedra join, sharing opposite faces with octahedra that separate them and (c) icosahedra join, sharing opposite edges with octahedra that separate them.

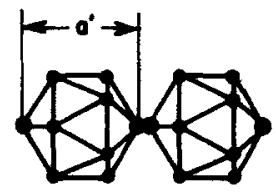

(a)

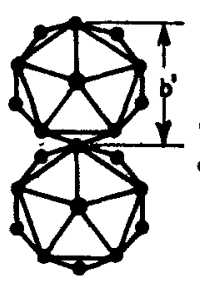

(b)

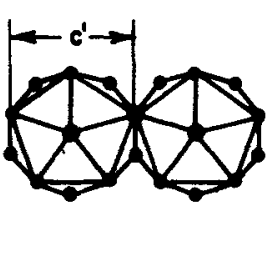

(c)

Figure 3. Emergence of the basic orthorhombic cell with parameters $a^{\prime}, b^{\prime}$ and $c^{\prime}$ by the coming together or linking of icosahedra in all three directions with (a) vertex, (b) face and (c) edge connections respectively. 


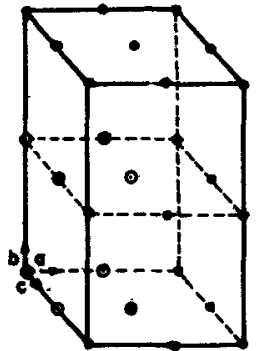

(a)

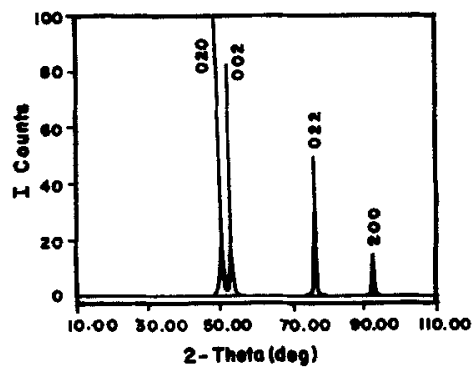

(b)

Figure 4. Refined basic orthorhombic cell, the building block for icosahedral phases, decagonal phases, and their rational approximants with (a) eight atomic positions, made up of two variants of four positions each and (b) computed powder diffraction intensities therefrom with equal partial occupancy by aluminium atoms only.

It has further been postulated by the author that the icosahedral, decagonal and rational approximant phases crystallize with large orthorhombic unit cells that are only superstructures of this small basic cell, built through complex ordering of dissimilar atoms and vacancies. If $a^{\prime}, b^{\prime}$ and $c^{\prime}$ are the parameters of the basic cell with 3 to 3.5 effective atoms, $m a^{\prime}, n b^{\prime}$ and $o c^{\prime}$ give the lattice parameters $a$, $b$ and $c$ respectively of the concerned orthorhombic cell, where $m, n$ and $o$ are integers. The values of these integers can generally be arrived at for a particular phase through careful analysis of its X-ray/neutron Debye-Scherrer patterns, particularly the positions of the few strong and medium reflections on the one hand and the many weak and very weak low-angle reflections on the other. In fact, as mentioned earlier, the weak reflections with the lowest Bragg angles offer important clues regarding the actual size of the unit cell. Incidentally, this family of orthorhombic crystals can also be indexed on a hexagonal or rhombohedral crystallographic framework, if so desired.

As explained earlier (Anantharaman 1989, 1990), the basic cell parameters $a^{\prime}$, $b^{\prime}$ and $c^{\prime}$ can be arrived at, approximately to start with, from the geometry of the ideal icosahedron whose edge length is taken as the average interatomic distance computed for the concerned alloy composition. This approach has led (Anantharaman 1994) to determination of the following lattice parameter values in the Al-Mn system: (a) For the icosahedral phase (i-Al-Mn): $a=6 a^{\prime}=15.288 \AA$; $b=4 b^{\prime}=17.376 \AA ; c=4 c^{\prime}=16.548 \AA$, (b) for the decagonal phase (d-Al-Mn): $a=6 a^{\prime}=15.258 \AA ; b=3 b^{\prime}=12.552 \AA ; c=4 c^{\prime}=16.418 \AA$ and (c) for some rational approximants of decagonal phases (including ternary alloys): $a=3 a^{\prime} ; b=3 b^{\prime} ; c=6 c^{\prime}$ (here the actual values will vary according to the chemical composition).

For many icosahedral phases, as also decagonal phases which display polytypism along the $b$ direction, excellent agreement between observed and calculated interplanar distances $\left(d_{h k l}\right)$ has been demonstrated, with strong and medium reflections showing appropriate indices like 200, 020, 002, 022 etc of the basic cell (Anantharaman 1993, 1994). Figure 4b shows the computed X-ray intensity values for the first 32 
powder reflections from the primitive basic cell (figure 4a) with only $\mathrm{Al}$ atoms in equal partial occupancy of the atomic sites. These data reflect a truly remarkable state of affairs, that has intrigued and confused crystallographers working in this field for many years. Thanks to the extraordinary symmetry and equal partial occupancy of the atomic sites, most reflections end up here with zero structure factor! It is most gratifying to note that only four X-ray reflections viz. 020, 002 , 022, and 200 in that order, make their appearance in the Bragg-angle range under consideration, the first two being the very strong reflections appearing close to each other and with an overlap in all icosahedral and decagonal phases respectively.

This model also explains many other features associated with these new phases. The nucleation problem gets solved, the growth of these phases starting homogeneously from icosahedral atomic clusters already existing in the alloy melts. The close relationship between icosahedral and decagonal phases is highlighted by the dimensions of their unit cells. In case of $i-\mathrm{Al}-\mathrm{Mn}$, despite the inevitable distortion due to linking of icosahedral clusters, the $b / c$ ratio remains at 1.05 , as in the ideal icosahedron. However, there seems to be more interpenetration and greater distortion of the icosahedron in the formation of $d-\mathrm{Al}-\mathrm{Mn}$. All the same, the observed epitaxial growth of $d$-Al-Mn on $i$-Al-Mn can be understood on the basis of their very close $a$ and $c$ parameters. A single space group viz. No. 47, has been assigned for the first time to all these phases. The extra atomic sites in the unit cells allow for maintenance of appropriate inter-atomic distances and the occurrence of a range in chemical composition. The five-fold diffraction symmetry gets explained in this icosahedral model through random changes in direction by $72^{\circ}$ during crystal growth and unusual orientation relationships (Bendersky et al 1989). Properties like hardness, brittleness, electrical resistivity etc of these phases can now be understood as due to each grain or crystal being a complex mosaic or irregularly-shaped and considerably inter-twined microcrystallites. A single crystal thus becomes a myth as far as these phases are concerned.

As an example, the $\mathrm{X}$-ray data obtained recently by the author at Dresden, Germany, on a rapidly solidified and annealed $i$-Al-Cu-Fe phase in a Siemans Rotating Anode X-ray Diffractometer are presented in table 2 in nanometer units. The agreement between calculation and observation is extremely good for all reflections, which were invariably sharp, except perhaps for the first weak reflection at a rather low Bragg angle, where the location of any peak is subject to uncertainties caused by the nature, size and shape of the specimen, its mounting pattern and related instrumental factors. It is noteworthy that all recorded reflections have indices ( $h k l$ values) common to both face-centred and body-centred cubic structures, as in case of the basic cell (figure $4 \mathrm{~b}$ ). As can be appreciated, once the extraordinary symmetry of the basic cell gets broken through complex ordering of dissimilar atoms and vacancies, all $\mathrm{X}$-ray reflections start appearing, albeit very weak in most cases, as from primitive orthorhombic structures.

The only problem yet to be sorted out concerns satisfactory matching of calculated and observed $\mathrm{X}$-ray/neutron intensities from $i-\mathrm{Al}-\mathrm{Cu}-\mathrm{Fe}$ and similar equilibrium icosahedral phases. As any crystallographer will readily concede, it is quite a challenge to place correctly so many atoms (around 288) belonging to three elements (Al, $\mathrm{Cu}$ and $\mathrm{Fe}$ in this case) in an ordered fashion with partial occupancy and vacant sites in a large superstructure (made up of 96 basic cells and with 576 possible atomic sites). However, it is only a matter of time, effort and appropriate computer software for this problem also to be solved satisfactorily. 
Table 2. Comparison of calculated and observed interplanar distances ( $d_{\mathrm{cal}}$ and $d_{\mathrm{obs}}$ in nanometers) and observed relative intensities $\left(I_{\mathrm{obs}}\right)$ for Debye-Scherrer reflections from Al-Cu-Fe icosahedral phase.

\begin{tabular}{rlllllllllr}
\hline No. & $h k l$ & $d_{\text {cal }}$ & $d_{\text {obs }}$ & $I_{\text {obs }}$ & No. & $h k l$ & $d_{\text {cal }}$ & $d_{\text {obs }}$ & $I_{\text {obs }}$ \\
\hline 1 & 020 & 0.842 & 0.833 & 2 & 17 & 624 & 0.204 & 0.204 & 2 \\
2 & 220 & $0.557 /$ & & & 18 & 008 & 0.200 & 0.200 & 100 \\
3 & 202 & 0.545 & 0.551 & 3 & 19 & 644 & 0.189 & 0.188 & 1 \\
4 & 040 & $0.421 /$ & & & 20 & 428 & 0.173 & 0.173 & 3 \\
5 & 004 & 0.401 & 0.415 & 3 & 21 & 448 & 0.163 & 0.162 & 2 \\
6 & 042 & 0.373 & 0.374 & 13 & 22 & 088 & 0.145 & 0.145 & 13 & 4 \\
7 & 420 & $0.340 /$ & & & 23 & 0.10 .6 & 0.143 & 0.142 & 1 \\
8 & 402 & 0.337 & 0.339 & 15 & 24 & 10.44 & 0.132 & 0.133 & 1 \\
9 & 224 & 0.325 & 0.324 & 12 & 25 & $22 \cdot 12$ & 0.130 & & \\
10 & 440 & 0.279 & 0.280 & 3 & 26 & 848 & 0.130 & 0.130 & 1 \\
11 & 600 & 0.248 & 0.245 & 7 & 27 & 12.00 & 0.124 & 0.124 & 36 \\
12 & 602 & 0.237 & 0.238 & 3 & 28 & 12.42 & 0.118 & 0.117 & 2 \\
13 & 444 & 0.229 & 0.229 & 2 & 29 & 12.80 & 0.107 & 0.107 & 20 \\
14 & 604 & 0.211 & & & 30 & 12.08 & 0.105 & & 15 \\
15 & 080 & 0.211 & 0.211 & 94 & 31 & 0.16 .0 & 0.105 & 0.105 & 15 \\
16 & 642 & 0.206 & 0.206 & 2 & 32 & 00.16 & 0.100 & 0.100 & 1 \\
\hline
\end{tabular}

Unit cell: Orthorhombic

Space group: $P_{\mathrm{mmm}}$ (No. 47)

$I_{\text {obs: }}$ : rounded to integers

$a=1.4868 \mathrm{~nm}$

$b=1.6840 \mathrm{~nm}$

$a=1.6024 \mathrm{~nm}$

(Weak reflections with $I_{\text {obs }}$ less than 1.0 are not recorded).

\section{The unified crystallographic approach}

Starting with icosahedral atomic clusters in alloy melts, that are made up of atoms of dissimilar sizes like those of aluminium and the transition metals, it is now possible to survey the whole field of complex phases in aluminium alloys and look for the inter-connections between the three major groups viz. (a) glassy or amorphous phases, (b) phases built with the orthorhombic basic cell, as described by the author, and (c) phases built with icosahedral clusters of different sizes, as visualized by Pauling.

The alloy melts may be expected to contain individual icosahedra made up of 13 or 12 atoms, separated from one another at higher temperatures. On cooling, these icosahedra may join together through their vertices to produce pentagonal atomic chains with ordering, that can stabilize the alloy melt. Such stabilization may lead to deep eutectics in the concerned alloy systems at compositions like $\mathrm{Al}_{12} \mathrm{~T}, \mathrm{Al}_{11} \mathrm{~T}, \mathrm{Al}_{11} \mathrm{~T}_{2}, \mathrm{Al}_{10} \mathrm{~T}_{2}, \mathrm{Al}_{10} \mathrm{~T}_{3}, \mathrm{Al}_{9} \mathrm{~T}_{3}, \mathrm{Al}_{9} \mathrm{~T}_{4}$ and $\mathrm{Al}_{8} \mathrm{~T}_{4}$ for binary alloys made up of aluminium and one transition metal $(T)$. The stabilization of the molten alloy may be easier for ternary and more complex alloys because of smaller atoms with different atomic radii becoming available for the ordering process. Rapid solidification of such alloys may lead to the formation of metallic glasses made up of individual icosahedra with random orientation and of broken, distorted and/or intertwined pentagonal chains, particularly in ternary and more complex aluminium alloys.

With decrease in cooling velocity, the icosahedral clusters and/or pentagonal chains in the melt may come together in all three directions to build up icosahedral, decagonal and the so-called rational approximant phases, as visualized by the author. 
Chemical composition, nature and number of alloying elements and actual cooling velocity - all will play a role in deciding the concerned orthorhombic structure (that can be viewed as hexagonal or rhombohedral as well) for these phases. Less imperfect and more stable phases seem to need higher transition metal content and two or more alloying elements as in case of $i-\mathrm{Al}-\mathrm{Cu}-\mathrm{Fe}, i-\mathrm{Al}-\mathrm{Cu}-\mathrm{Ru}$ and $d-\mathrm{Al}-\mathrm{Cu}-\mathrm{Co}$. The decagonal phases may display different stacking sequences in one direction i.e. show polytypism, with stacking faults in some cases. It is attractive to consider all these as forming a special group of 'Hume-Rothery phases', even though the criteria are yet to be clearly understood and satisfactorily worked out for such an eventuality.

With normal cooling, individual and separate icosahedra may build up cubic, especially body-centred cubic (bcc) structures. Taking, as the first example, $\mathrm{Al}_{12} \mathrm{~W}$, $\mathrm{Al}_{12} \mathrm{Mo}, \mathrm{Al}_{12} \mathrm{Cr}, \mathrm{Al}_{12} \mathrm{Mn}, \mathrm{Al}_{12} \mathrm{Re}$, etc with two 13-atom clusters, the cluster at the origin gets linked to the cluster at the body centre by an octahedron, sharing opposite faces with both (figure 5a). Accordingly, the sequence along the body diagonal in this bcc structure is A, A, A ... (see figure 2b). Bigger icosahedral clusters may also form, starting from the 13- or 12-atom icosahedra, and in fact, have been identified according to their sizes as Mackay icosohedra, 'soccer ball' etc. These also lead to bcc structures, the best known among them being the Frank-Kasper or T-phases containing 162 atoms. It was shown by Cenzual et al (1985) that the T-phase clusters can also be described in terms of linking or packing of icosahedra (see figure 5b). In this approach a shell of 12 centred icosahedra is created through edge-sharing, with the 12 inner vertices of these icosahedra forming an inner icosahedron with its central atom missing. The 13 icosahedra all have the same spatial orientation. The central icosahedron has to be somewhat smaller than the outer ones, just as the central atom has to be smaller than the ones forming the central icosahedron. This latter circumstance may well be the reason for the suspected absence of the central atom in $\mathrm{T}$-phase structures, as also in $\alpha$-( $\mathrm{Al}$, $\mathrm{Si})_{57} \mathrm{Mn}_{12}$ and related phases (see Guyot and Audier 1985). In all such bcc structures, in principle, it should be possible to arrive at the lattice parameters approximately, as in the author's model for icosahedral and decagonal phases, from the geometry of the inner icosahedron and the outer shells. In fact, the actual nature of the linkage between icosahedra may well be settled through such geometrical considerations.

To sum up, the golden thread running through the numerous Al-TM complex phases viz. metallic glasses, icosahedral phases, decagonal phases, rational crystalline approximants, Frank-Kasper and other equilibrium phases, can now be identified as the icosahedral atomic cluster present in all Al-TM alloy melts. Starting from such clusters, the crystalline phases form through homogeneous nucleation from them in two ways, one leading to orthorhombic, hexagonal and rhombohedral structures and the other to cubic structures, while the metallic glasses form by the freezing-in of individual clusters and vertex-joined pentagonal chains of atoms from the liquid state. The chemical composition of the alloy, as also the relative sizes and electronic structures of the different atoms in it, decide the mode of solidification under equilibrium conditions i.e. slow cooling from the melt. On the other hand, the high rates of cooling in rapid solidification may modify and change this mode drastically to produce different non-equilibrium phases with unusual $72^{\circ}$ orientation relationships and complex interfaces between the microcrystallites that generally 


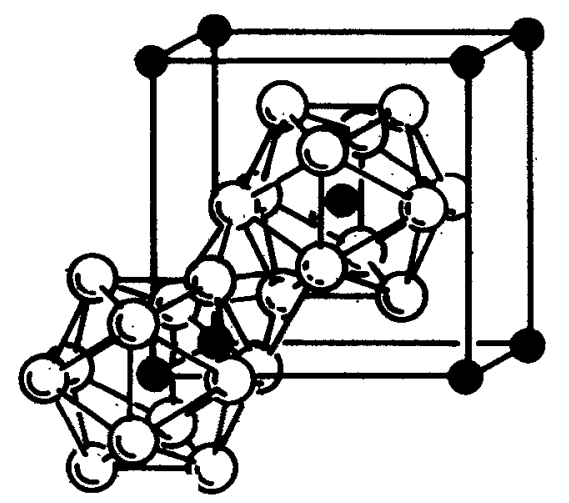

(0)

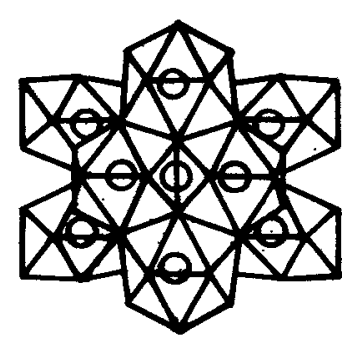

(b)

Fgure 5. (a) Unit cell of $\mathrm{Al}_{12} M$ (when $M=\mathrm{W}, \mathrm{Mo}, \mathrm{Cr}, \mathrm{Re}$ or $\mathrm{Tc}$ ) with 13-atom clusters at origin and body centre linked by an octahedron sharing opposite faces, (b) cluster of 12 icosahedra sharing edges to define a larger icosahedron in Frank-Kasper or T-phases. One may note the distortions necessitated by the close approaches of atoms in icosahedra that are joined by edge-sharing.

constitute every grain in such polycrystalline materials. Needless to say, further accurate experimentation, painstaking analysis of available data and sophisticated computational procedures will all be needed to confirm this unified crystallographic approach.

In the author's view, Pauling grasped intuitively, rather than through a logical and cugent thinking process, the structural unity underlying complex crystalline and so-called quasicrystalline phases in aluminium alloys. He could not possibly put it across effectively, even though he authored many papers, as he worked practically alone in this area, depended heavily on the experimental data of others and also faced considerable hostility from the highly vocal and hyperactive leaders on the quasicrystallography bandwagon. His anger and anguish at the early turn of events led to some strong pronouncements e.g. 'this nonsense about quasicrystals' and 'I have difficulty getting my papers on the subject published' (Pauling 1986). He was seemingly on the right track, however, and his assertion in an interview that 'history is on my side' (Pauling 1993) may well prove to be prophetic. 


\section{References}

Anantharaman T R (ed.) 1984 Metallic glasses: production, properties and applications (Switzerland: Trans Tech Publications)

Anantharaman T R 1987 Mater. Sci. Forum 22-24 55

Anantharaman T R 1988a Curr. Sci. 57578

Anantharaman T R 1988b Scr. Metall. 22981

Anantharaman T R 1989 Curr. Sci. 581067

Anantharaman T R 1990 in Quasicrystals and incommensurate structures in condensed matter (od.) M Jose Yacaman, D Romeu, V Castano and A Gomez (Singapore: World Scientific) p. 199

Anantharaman T R 1993 Scr. Met. Mater. 281555

Anantharaman T R 1994 in Experimental methods of phase diagram determination (ed.) J E Morral, R $S$ Schiffman and $\$$ M Merchant (USA: TMS) p. 173

Anantharaman T R 1994a Philos. Mag. Lett. (communicated)

Anantharaman T R 1994b Scr. Met. Mater. (communicated)

Anantharaman T R and Adhavan E 1994 Trans. Indian lnst. Met. 4767

Anantharaman T R, Ramachandrarao P, Suryanarayana C, Lele S and Chattopadhyay K 1977 Trans. Indian Inst. Met. 30 423, 434

Anantharaman T R and Suryanarayana C 1987 Rapidly solidified metals: A technological overview (Switzerland: Trans. Tech. Publications)

Ball M D and Lloyd D J 1985 Scr. Metall. 191065

Bancel P A, Heiney P A, Stephens P W and Goldman A I 1986 Nature (London) 319104

Bendersky A, Cahn J W and Gratias D 1989 Philos. Mag. BCo 837

Bergmar G, Waugh J L T and Pauling L 1957. Acta Crystallogr. 10254

Bland J A 1958 Acta Crystallogr. 11236

Cahn J'W, Gratias D and Schechtman D 1986 Nature (London) 319102

Cart M J 1985 J. Appl. Phys. 591063

Cenzual K, Chabot B and Parthe E 1985 Acta Crystallogr. C41 313

Fettweis M, Launois P, Denoyer F, Reich R, Godard J M and Lambert M 1993 J. Non-Cryst. Solids 153/154 595

Fettweis M, Launois P, Dénoyer F, Reich R and Lambert M 1994 Phys. Rev. B49 15573

Field R D and Fraser H L 1985 Mater. Sci. Eng. 68 L17

Frank F C 1952 Proc. R. Soc. London A215 HLA3

Goldman A I and Kelton K F 1993 Rev. Modern Phys. 65213

Guyot P and Audier M 1985 Philos. Mag. B52 L15

Hume-Rothery W and Anderson E 1960 Philos. Mag. 5383

Janot C 1993 Proc. $R$. Soc. London A442 113

Levine D and Steinhardt P J 1984 Phys. Rev. Lett. 532477

Liu W 1993 Fortschritt-Berichte VDI Reihe 5 No 300, pp. 9 and 70

Mackay A L 1982 Physica (Amsterdam) A114 609

Mackay A L 1986 Nature (London) 319103

Motech T, Denoyer F, Launois P and Lambert M 1992 J. Phys. I. France 2861

Pauling L 1955 Am. Sci. 43285

Pauling L 1985 Nature (London) 317512

Pauling L 1986 Science News 1293

Pauling L 1987a Phys. Rev. Lett. 58365

Pauling L 1987 Proc. Natl. Acad. Sci. USA 843537

Pauling L 1987c Proc. Natl. Acad. Sci. USA 843951

Pauling L 1988a Proc. Natl. Acad. Sci. USA 852422

Pauling L 1988b Proc. Natl. Acad. Sci. USA 853666

Pauling L 1988c Proc. Natl. Acad. Sci. USA 854587

Pauling L 1988d Proc. Natl. Acad. Sci. USA 858376

Pauling L 1989a Proc. Natl. Acad. Sci. USA 868595

Pauling L 1989b Proc. Natl. Acad. Sci. USA 869637

Pauling L 1990 Proc. Natl. Acad. Sci. USA 877849

Pauling L 1991 Proc. Natl. Acad. Sci. USA 886600

Pauling L 1993 in John Horgan's Profile, Scientific American 268-3 36 
Rao V V and Anantharaman T R 1988 Mater. Sci. Eng. 99393

Schechtman B and Blech I A 1985 Mater. Trans. A16 1005

Schechtman D, Blech I, Gratias D and Cahn J W 1984 Phys. Rev. Lett. 531951

Shoemaker D P and Shoemaker C B 1988 in Introduction to quasicrystals (ed.) M Jaric (Academic Press) p. 1

Stephens P W and Goldman A I 1986 Phys. Rev. Lett. 561168

Steurer W 1990 Z Kristallogr. 190179

Vecchio K S and Williams D B 1988a Metall. Trans. A19 2875

Vecchio K S and Williams D B 1988b Philos. Mag. B57 535 